\title{
Therapy-related myeloid neoplasms following fludarabine, cyclophosphamide, and rituximab (FCR) treatment in patients with chronic lymphocytic leukemia/small lymphocytic lymphoma
}

\author{
Yi Zhou ${ }^{1}$, Guilin Tang ${ }^{1}$, L Jeffrey Medeiros ${ }^{1}$, Timothy J McDonnell ${ }^{1}$, Michael J Keating ${ }^{2}$, \\ William G Wierda ${ }^{2}$ and Sa A Wang ${ }^{1}$ \\ ${ }^{1}$ Department of Hematopathology, The University of Texas, MD Anderson Cancer Center, Houston, TX, USA \\ and ${ }^{2}$ Department of Leukemia, The University of Texas, MD Anderson Cancer Center, Houston, TX, USA
}

\begin{abstract}
This study is focused on therapy-related myeloid neoplasms after the most promising frontline FCR (fludarabine, cyclophosphamide, and rituximab) therapy in previously untreated chronic lymphocytic leukemia patients. A total of 28 therapy-related myeloid neoplasm patients were identified, including 19 patients from 3 well-controlled FCR frontline trials $(n=426$ patients), giving an estimated frequency of $4.5 \%(1.9-8.3 \%)$ in a follow-up period of 44 months (range 5-122 months). Clinically, therapy-related myeloid neoplasms could emerge directly from 'prolonged myelosuppression' after FCR (10 patients), or after achieving complete hematological recovery $(n=18)$. The overall latency was 35 months (range 3-118 months), with the former group of 23 months and the latter 42 months $(P<0.001)$. In all, 10 cases presented as therapy-related acute myeloid leukemia and 18 as therapy-related myelodysplastic syndromes. Abnormal cytogenetics was present in 26 of $27(96 \%)$ patients, with frequent chromosomes 5 and 7 abnormalities. The median survival was 7 months after therapy-related myeloid neoplasms. Our results indicate that the risk of therapy-related myeloid neoplasms secondary to frontline FCR therapy may not be as high as previously reported after removing the confounding factor of previous cytotoxic exposure, but this risk increased with older age and likely growth factor co-administration. Therapy-related myeloid neoplasms after FCR therapy shares clinicopathological features with therapy-related myeloid neoplasms secondary to other alkylating agents, but has a shorter latency interval indicating possible synergetic effects of the nucleotide analog fludarabine. The fact that therapy-related myeloid neoplasms can directly emerge from 'prolonged myelosuppression' warrants a bone marrow examination to rule out therapy-related myeloid neoplasms in this clinical setting.

Modern Pathology (2012) 25, 237-245; doi:10.1038/modpathol.2011.158; published online 11 November 2011
\end{abstract}

Keywords: chronic lymphocytic leukemia; cytogenetics; FCR; survival; therapy-related myeloid neoplasm

Therapy-related myeloid neoplasms as a result of exposure to chemotherapy or ionizing radiation are infrequent, but this therapeutic complication is associated with dismal patient outcome. In general, therapy-related myeloid neoplasms secondary to

Correspondence: Associate Professor SA Wang, MD, Department of Hematopathology, University of Texas M. D. Anderson Cancer Center, Box 72, 1515 Holcombe Boulevard, Houston, Texas 770304009, USA.

E-mail: swang5@mdanderson.org

Received 19 July 2011; revised 24 August 2011; accepted 26 August 2011; published online 11 November 2011 alkylating agents or ionizing radiation occur approximately 5-10 years after exposure and often present as therapy-related myelodysplastic syndromes. By contrast, therapy-related myeloid neoplasms after DNA topoisomerase II inhibitor therapy often have a median duration of 1-5 years and more commonly present as therapy-related acute myeloid leukemia. ${ }^{1}$ The former patient group presents with a gradual progression of cytopenia and is commonly associated with unbalanced loss of genetic material, most often involving chromosomes 5 and/or 7. The latter patient group presents with overt acute leukemia and is commonly associated with 
balanced chromosomal translocations. In practice, therapy-related myeloid neoplasms in relation to its causal agents are difficult to characterize, as most patients receive multiple cytotoxic agents over lengthy treatment for primary tumors, either due to refractory disease or disease progression. The frequencies and clinical presentation reported are variable among different treatment regimes for different types of cancers. ${ }^{2-4}$ Nevertheless, knowledge of the clinical presentation and genetic features of therapy-related myeloid neoplasms that are associated with a particular chemotherapy regimen is important for earlier diagnosis of therapy-related myeloid neoplasms, appropriate patient management, and possibly selection of further therapies.

Chronic lymphocytic leukemia/small lymphocytic lymphoma is the most common type of leukemia in adults of Western countries. Patients with chronic lymphocytic leukemia/small lymphocytic lymphoma usually have an indolent disease course, but untreated progressive disease is inevitably fatal. The outcome of chronic lymphocytic leukemia/small lymphocytic lymphoma, especially in the younger patient group, had been significantly improved from recent advances in chemotherapy. The combination regimen, composed of the purine analog fludarabine (F), the alkylating agent cyclophosphamide (C), and the humanized anti-CD20 monoclonal antibody rituximab (R), has emerged as a highly effective frontline therapy for chronic lymphocytic leukemia/small lymphocytic lymphoma patients. Previous studies have demonstrated a complete remission rate of $72 \%$, and 6-year failure-free survival of $51 \% .^{5}$ The most important toxicity of the FCR regimen is myelosuppression, causing serious neutropenia (absolute neutrophil count $<1000 / \mu \mathrm{l}$ ) in $>50 \%$ treatment courses. ${ }^{6}$ Most patients recover within a short period of time, but prolonged cytopenia ( $>3$ months) can occur in 19$43 \%$ of patients at the end of the treatment. ${ }^{5,7}$ Although cytopenia can usually be attributed to bone marrow injury and blood counts eventually recover, other causes of cytopenia, including autoimmune hemolytic anemia and therapy-related myeloid neoplasms, need to be excluded. Autoimmune cytopenia, either positive or negative for direct antiglobulin test, has been seen in chronic lymphocytic leukemia/small lymphocytic lymphoma patients after fludarabine containing chemotherapy, ${ }^{8,9}$ for which steroid and cyclosporine treatment has shown some efficacy. ${ }^{9}$ Therapy-related myeloid neoplasms, including therapy-related myelodysplastic syndrome and therapy-related acute myeloid leukemia, are much less common, but have an ominous outcome. Therapy-related myeloid neoplasms after FCR therapy have been reported in anecdotal cases ${ }^{10-12}$ or in case series. ${ }^{13-16}$ However, most of those patients have been previously treated with non-FCR cytotoxic agents; therefore, therapyrelated myeloid neoplasms in those settings more likely reflect an accumulated cytotoxic exposure.
Furthermore, the clinicopathological features and genetic characteristics of therapy-related myeloid neoplasms after FCR treatment in chronic lymphocytic leukemia/small lymphocytic lymphoma patients remain to be fully elucidated.

In this study, we studied therapy-related myeloid neoplasms arising in chronic lymphocytic leukemia/small lymphocytic lymphoma patients who received FCR as the frontline chemotherapy regimen. This study group allowed us not only to assess the real risk of therapy-related myeloid neoplasms in chronic lymphocytic leukemia/small lymphocytic lymphoma patients treated with FCR but also to better characterize the clinicopathological and cytogenetic features of therapy-related myeloid neoplasms arising in this setting, information critical for an accurate early diagnosis and management of patients.

\section{Materials and methods}

\section{Patients}

This is a retrospective study based on the review of patient charts in a single institution. All included patients had untreated chronic lymphocytic leukemia/small lymphocytic lymphoma before receiving FCR, and subsequently developed secondary myeloid neoplasm after FCR treatment. This study was initially focused on 3 FCR frontline clinical trials, which enrolled a total of 426 patients. These well-controlled trials allowed us to study the risk of therapy-related myeloid neoplasms associated with FCR therapy alone. In addition, to better characterize the clinicopathological features of therapyrelated myeloid neoplasms arising in this setting, we also searched the pathology data files between 2002 and 2010 for additional cases. All therapy-related myeloid neoplasms were confirmed by pathological assessment, in conjunction with cytogenetic findings and clinical follow-up data. Patients were excluded from this study if myeloid neoplasm was diagnosed before or at the same time as chronic lymphocytic leukemia/small lymphocytic lymphoma or patients had received chemotherapy other than FC/FCR for chronic lymphocytic leukemia/small lymphocytic lymphoma before the diagnosis of therapy-related myeloid neoplasms. As this study was a retrospective study, no additional patient consent was obtained. This study is approved by the Institutional Review Board.

\section{FCR Treatments}

The detailed FCR treatment plan has been published previously. ${ }^{5}$ In brief, patients received rituximab (375$\left.500 \mathrm{mg} / \mathrm{m}^{2}\right)$ on day 1 , and fludarabine $\left(25-30 \mathrm{mg} / \mathrm{m}^{2}\right.$ daily) and cyclophosphamide (250-300 $\mathrm{mg} / \mathrm{m}^{2}$ daily) on days 1-3 of each course (days 2-4 for the first course only). Treatment was repeated every 4 weeks 
for a total of 6 courses. The treatment course would be delayed or stopped if the platelet count was lower than $80 \times 10^{9} / 1$ and the absolute neutrophil count was lower than $1 \times 10^{9} / 1$. Consents were obtained from all patients for receiving FCR treatment.

\section{Laboratory Data and Bone Marrow Assessment}

Laboratory data for patients in the study group, including complete blood count, cytogenetic analysis, and bone marrow morphology, were retrieved and correlated with the clinical course of chronic lymphocytic leukemia/small lymphocytic lymphoma progression, treatment, and development of therapy-related myeloid neoplasms. All chronic lymphocytic leukemia/small lymphocytic lymphoma and therapy-related myeloid neoplasm diagnostic materials were obtained and reviewed at our institution. Patients who presented with reversible cytopenia(s), transient bone marrow morphological dysplasia, or transient cytogenetic abnormalities were excluded after reviewing follow-up clinical laboratory data.

In this study, cytopenia is defined as neutrophils $<1.5 \times 10^{9} /$, platelet $<100 \times 10^{9} / 1$, or hemoglobin $<11.0 \mathrm{~g} / \mathrm{dl}$ without exogenous growth factor treatment. ${ }^{17}$ Representative bone marrow aspirate smears and trephine biopsy specimens were available for evaluation of all included patients. A 500 cell differential count was performed based on examination of multiple fields of bone marrow aspirate smears, and 200 cell counts on peripheral blood smears if pathological cells were reported by an automatic hematology analyzer. Myeloblasts were enumerated as a percentage of total bone marrownucleated cells in myelodysplastic syndrome and acute myeloid leukemia, and of non-erythroid elements in acute erythroleukemia (M6a). For the diagnosis of morphological dysplasia, features of dyserythropoiesis, dysgranulopoiesis, and dysmegakaryopoiesis had to be present in $\geq 10 \%$ of cells of the respective lineage, as specified in the 2008 WHO classification. ${ }^{1}$ Iron stain was performed on all available bone marrow aspirate smears to assess storage iron and ring sideroblasts. If fibrosis was suspected on biopsy, reticulin and Massontrichrome stains were performed to evaluate the degree of fibrosis.

\section{Cytogenetics}

Conventional cytogenetic analysis was performed using standard methods. In all, 20 metaphases were analyzed in all cases, if available, and the results were reported using the International System for Human Cytogenetic Nomenclature. In some cases, a lesser number of metaphases was available, but in all cases, the numbers of metaphases were adequate to issue a karyotype. FISH analysis for chronic lymphocytic leukemia/small lymphocytic lymphoma was performed using a multicolor probe panel designed to provide simultaneous detection of the 11q22.3 (ATM gene) region of chromosome 11, the 17p13.1 (TP53 gene) region of chromosome 17, the $\alpha$-satellite, centromeric region of chromosome 12 (D12Z3), the D13S319 locus (located between RB1 and D13S25 loci) in the $13 q 14.3$ region of chromosome 13 , and the $13 q 34$ region (LAMP1 gene) near the subtelomere of chromosome 13q (Abbott Molecular). FISH analysis for deletion of chromosomes 5 and 7 was performed using a Vysis LSI EGR1/D5S23, D5S721 dual-color probe and a CEP $\alpha$-satellite (D7Z1) 7-probe, respectively, in a subset of cases (Vysis, Downers Grove, IL). A total of 200 interphases were analyzed for each probe.

\section{Statistical Analysis}

Fisher's exact test was applied for categorical variables. Patient survival was estimated by the Kaplan-Meier method from the date of therapyrelated myeloid neoplasm diagnosis until death attributed to therapy-related myeloid neoplasms (disease-specific survival). Patients who were alive at the latest follow-up were censored. Survival curves were statistically compared by the log-rank test. Differences between two groups were considered statistically significant if $P$-values were $<0.05$ in a two-tailed test.

\section{Results}

\section{Patient Characteristics}

In all, 19 patients ${ }^{18}$ who developed therapy-related myeloid neoplasms after frontline FCR therapy were identified from the 3 well-controlled clinical trials that enrolled a total of 426 patients. These 3 trials included 1 standard frontline FCR trial consisting of 300 patients, 1 FCR plus concurrent GM-CSF consisting of 60 patients, and 1 FC plus multiple cycles of rituximab consisting of 66 patients. To better characterize the clinicopathological features of therapy-related myeloid neoplasms, additional 9 patients were identified from our pathology data files consisting of $\sim 3000$ chronic lymphocytic leukemia/small lymphocytic lymphoma patients. These nine patients were treated with FCR frontline off protocol at our hospital. The characteristics of these patients are listed in Table 1 . There were 21 men and 7 women with a median age of 67 years (range 47-80 years). Eight of these patients later received FCR salvage therapy with or without additional immunotherapy for recurrent chronic lymphocytic leukemia/small lymphocytic lymphoma. The median follow-up period interval including alive and dead patients was 44 months (range 5-122 months). 
Table 1 Status of chronic lymphocytic leukemia/small lymphocytic lymphoma in 28 patients who later developed therapyrelated myeloid neoplasm

\begin{tabular}{|c|c|}
\hline Patients $(n)$ & 28 \\
\hline Age (years, median (range)) & $67(47-80)$ \\
\hline Gender (male/female) ( $n$ ) & $21 / 7$ \\
\hline CLL vs SLL presentation $(n)$ & 25 and 3 \\
\hline Total follow-up (months) & $44(5-122)$ \\
\hline$\beta$-2 Microglobulin (mg/l) & $4.0(1.9-10.0)$ \\
\hline LDH (IU/l) & $600(336-2232)$ \\
\hline Bone marrow involvement by CLL/SLL (\%) & $80(70-100)$ \\
\hline \multicolumn{2}{|l|}{ Cytogenetic analysis (n) } \\
\hline Diploid karyotype & 13 \\
\hline Abnormal karyotype & 12 \\
\hline \multicolumn{2}{|l|}{ FISH analysis (n) } \\
\hline del (ATM) & 5 \\
\hline del (D13S319) & 4 \\
\hline Trisomy 12 & 4 \\
\hline $\operatorname{del}(11 q 14)$ & 3 \\
\hline \multicolumn{2}{|l|}{ Treatment response to FCR (n) } \\
\hline \multicolumn{2}{|l|}{ FCR cycles } \\
\hline Completed six cycles & 20 \\
\hline Less than six cycles & 8 \\
\hline Complete remission (CR) & 12 \\
\hline Complete remission with incomplete & 14 \\
\hline \multicolumn{2}{|l|}{ bone marrow recovery (CRi) } \\
\hline Persistent cytopenia & 10 \\
\hline Recovered CBC later & 4 \\
\hline Partial remission & 2 \\
\hline
\end{tabular}

Chronic Lymphocytic Leukemia/Small Lymphocytic Lymphoma Status of Study Group before FCR Treatment

The chronic lymphocytic leukemia/small lymphocytic lymphoma status at the time of initiation of FCR treatment is summarized in Table 1. Bone marrow biopsy of 24 patients performed before FCR treatment showed a median chronic lymphocytic leukemia infiltrate of $80 \%$, ranging from 70 to nearly $100 \%$. The median $\beta$-2-microglobulin was $4 \mathrm{mg} / \mathrm{l}$ and 13 patients had LDH level >600 IU/l. In all, 25 patients had conventional cytogenetic analysis and 8 patients had FISH performed before FCR therapy, respectively. Overall cytogenetic abnormalities were detected in 15 cases, including 4 trisomy 12, 5 partial deletion of chromosome 13q (D13S319), and 5 loss of ATM locus.

\section{Frequency of Therapy-Related Myeloid Neoplasms after FCR Treatment}

The risks of therapy-related myeloid neoplasms after FCR treatment were estimated from 426 chronic lymphocytic leukemia/small lymphocytic lymphoma patients enrolled in 3 FCR-related clinical trials. In the standard frontline FCR trial that enrolled 300 patients, 10 patients developed therapy-related myeloid neoplasms. Among them, 3 out of 186 patients $(1.9 \%)$ were younger than 60 years and 7 out of
$114(6.1 \%)$ patients were $\geq 60$ years; the difference was statistically significant $(P=0.046)$. In the other 2 trials, 5 out of 60 patients (median age 67 years) treated with FCR plus concurrent GM-CSF (8.3\%), and 4 out of 66 patients (median age 68 years) treated with FC plus multiple cycles of rituximab $(6.1 \%)$ developed therapy-related myeloid neoplasms. The overall risk of therapy-related myeloid neoplasms within the follow-up period was $4.5 \%$. The remaining nine patients were treated off protocol, and the risk was difficult to assess.

\section{Therapy-Related Myeloid Neoplasms}

The characteristics of therapy-related myeloid neoplasms in the 28 patients are summarized in Table 2. In all, 20 patients ${ }^{19}$ completed standard 6-cycle FCR treatment, whereas 8 patients aborted the treatment after 5 cycles because of prolonged cytopenia. After FCR therapy, $12(43 \%)$ patients achieved complete remission, $14(50 \%)$ achieved complete remission with incomplete bone marrow recovery, and 2 achieved partial remission. In all, 10 of the 14 patients with complete remission with incomplete marrow recovery had persistent cytopenia until the diagnosis of therapy-related myeloid neoplasms. The others had recovered blood counts after FC/ FCR, and later developed cytopenia. The median duration from initiation of FCR treatment to diagnosis of therapy-related myeloid neoplasms of the whole group was 35 months (range 3-118 months). In 10 patients who never completely recovered their blood count after FC/FCR, the median duration to therapy-related myeloid neoplasms was 23 months (range 3-43 months), significantly shorter than 42 months (range 24-118 months) for the 18 patients who had normalized blood counts and later developed cytopenia $(P<0.01)$ (Figure 1$)$. It is noteworthy that 5 out of 7 patients who had latency interval $>60$ months had received additional FCR treatment for recurrent chronic lymphocytic leukemia/small lymphocytic lymphoma.

At the time of therapy-related myeloid neoplasms diagnosis, 17 (61\%) patients had no morphological or immunophenotypic evidence of chronic lymphocytic leukemia/small lymphocytic lymphoma in the bone marrow, 11 (39\%) patients had recurrent/persistent chronic lymphocytic leukemia/ small lymphocytic lymphoma, whereas chronic lymphocytic leukemia/small lymphocytic lymphoma cells comprised $<10 \%$ bone marrow cellularity in 6 patients, and only 1 patient had significant chronic lymphocytic leukemia involvement in the bone marrow $(80 \%)$. The median bone marrow cellularity was $40 \%$ (range $20-90 \%$ ). According to 2008 WHO criteria, 5 (18\%) cases were therapyrelated acute myeloid leukemia and 23 cases of therapy-related myelodysplastic syndromes. Of the therapy-related myelodysplastic syndrome, 8 (29\%) cases were refractory cytopenia with excessive 
Table 2 Characterization of therapy-related myeloid neoplasms

\begin{tabular}{|c|c|}
\hline Latency (months) & $35(3-118)$ \\
\hline $\begin{array}{l}\text { Patients with persistent cytopenia } \\
\text { after FCR }(n=10) \text { (months) }\end{array}$ & $23(3-43)$ \\
\hline $\begin{array}{l}\text { Patients recovered CBC after FCR } \\
\text { and later developed cytopenia } \\
(n=18) \text { (months) }\end{array}$ & $42(24-118)$ \\
\hline \multicolumn{2}{|l|}{ Cytopenia (at diagnosis of $t-M N$ ) } \\
\hline Anemia & $n=18$ \\
\hline Hemoglobin (g/dl) & Median/range 10 (6.6-15.7) \\
\hline Thrombocytopenia & $n=19$ \\
\hline Platelets $\left(\times 10^{9} / 1\right)$ & Median/range 45.5 (7-388) \\
\hline Neutropenia & $n=16$ \\
\hline $\begin{array}{l}\text { Absolute neutrophil } \\
\text { count (ANC) }\left(\times 10^{9} / 1\right)\end{array}$ & Median/range 1.1(0.2-5.9) \\
\hline Bicytopenia & $n=10$ \\
\hline Pancytopenia & $n=9$ \\
\hline \multicolumn{2}{|l|}{ Bone marrow } \\
\hline Cellularity (\%) & $40(20-90)$ \\
\hline \multicolumn{2}{|l|}{ Dysplasia } \\
\hline Trilineage dysplasia & $n=18$ \\
\hline Bilineage dysplasia & $n=3$ \\
\hline Single lineage dysplasia & $n=4$ \\
\hline Ring sideroblasts & $\begin{array}{c}n=12(2-46 \%) \text { and } \geq 15 \% \\
\text { in } 8 \text { cases }\end{array}$ \\
\hline \multicolumn{2}{|l|}{ CLL/SLL in bone marrow } \\
\hline Negative & $n=17$ \\
\hline$\leq 10 \%$ & $n=6$ \\
\hline$>10 \%$ & $n=5$ \\
\hline \multicolumn{2}{|l|}{ t-MN subtypes by WHO criteria } \\
\hline t-AML & $n=5$ \\
\hline t-MDS & $n=23$ \\
\hline RAEB-1 & $n=5$ \\
\hline RAEB-2 & $n=3$ \\
\hline RCMD & $n=11$ \\
\hline RARS & $n=2$ \\
\hline MDS-U & $n=2$ \\
\hline \multicolumn{2}{|l|}{ Cytogenetics } \\
\hline Diploid & $n=1$ \\
\hline Abnormal karyotype & $n=26$ \\
\hline$-5 / \operatorname{del}(5 q)$ and $/$ or $-7 / \operatorname{del}(7 q)$ & $n=18$ \\
\hline Complex karyotype & $n=2$ \\
\hline Trisomy 8 & $n=3$ \\
\hline$-20 / \operatorname{del}(20 q)$ & $n=2$ \\
\hline Disease-specific survival (months) & Median $=7(1-22)$ \\
\hline
\end{tabular}

MDS-U, myelodysplastic syndromes-unclassifiable; RAEB, refractory anemia with excess blasts; RCMD, refractory cytopenia with multilineage dysplasia; t-AML, therapy-related acute myeloid leukemia; tMDS, therapy-related myelodysplastic syndromes; therapy-related myeloid neoplasms: therapy-related myeloid neoplasms.

blasts (5 refractory anemia with excess blasts-1 and 3 refractory anemia with excess blasts-2), 11 cases of refractory cytopenia with multilineage dysplasia, 2 refractory anemia with ring sideroblasts, and 2 myelodysplasia, unclassifiable. Morphologically, therapy-related myelodysplastic syndrome secondary to FCR frequently had a low or normal cellularity with relative marked erythroid hyperplasia/predominance (Figure 2). Dysplasia-involving two or more lineages was common; and ring sideroblasts were present in variable numbers $(2-46 \%)$ in 12 cases (Table 2) and $\geq 15 \%$ blasts in 8 cases.

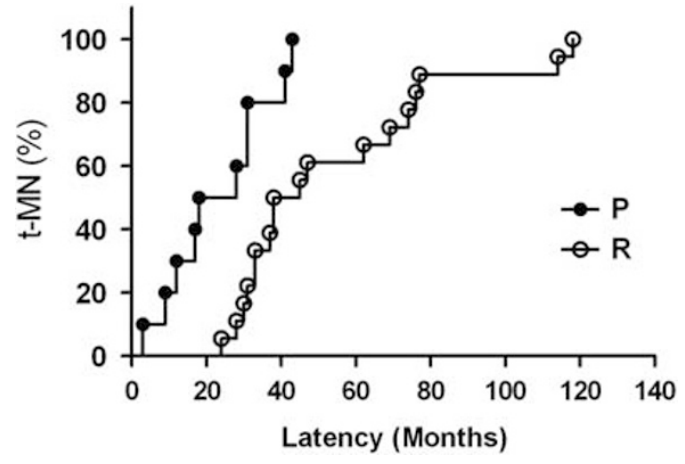

Figure 1 Latency intervals of therapy-related myeloid neoplasms after FCR treatment were estimated by the Kaplan-Meier curves. P: patients presented with a clinical picture of 'prolonged myelosuppression' after FCR; R: patients achieved a complete hematological recovery after FCR, but later developed therapy-related myeloid neoplasms. The latency intervals were significantly different between these two groups of patients (23 vs 42 months, $P<0.001)$.

Post-treatment changes, such as stromal edema/ serous changes, increased histiocytes, plasma cells, and mast cells, were observed in a number of cases, especially in cases in which therapy-related myelodysplastic syndrome directly arose from 'myelosuppression'. Cytogenetic abnormalities were observed in 26 of 27 (96\%) cases with available cytogenetic result at the time of therapy-related myeloid neoplasms diagnosis: 18 (67\%) cases had unbalanced partial or complete loss of chromosome 7 and/or chromosome 5. The remaining 8 cases included 2 with a complex karyotype, 3 with trisomy 8 , and 2 with partial or complete loss of chromosome 20. None of the cases showed 11q23 abnormalities.

\section{Prognosis of FCR-Related Therapy-Related Myeloid Neoplasms}

Patients received various treatments after diagnosis of therapy-related myeloid neoplasms, including induction chemotherapy for therapy-related acute myeloid leukemia, hypomethylation agent for therapy-related myelodysplastic syndrome, transfusions, and supportive care. Five patients with therapy-related myelodysplastic syndrome evolved into therapy-related acute myeloid leukemia. Six patients underwent stem cell transplant. With a median follow-up of 8 months including alive and dead, $21(75 \%)$ patients died of therapy-related myeloid neoplasms with a median disease specific survival of 7 months (Figure 3). Three of six patients who underwent stem cell transplant were alive at the time of last follow-up.

\section{Discussion}

In this study, we identified 28 chronic lymphocytic leukemia/small lymphocytic lymphoma patients who developed therapy-related myeloid neoplasms 


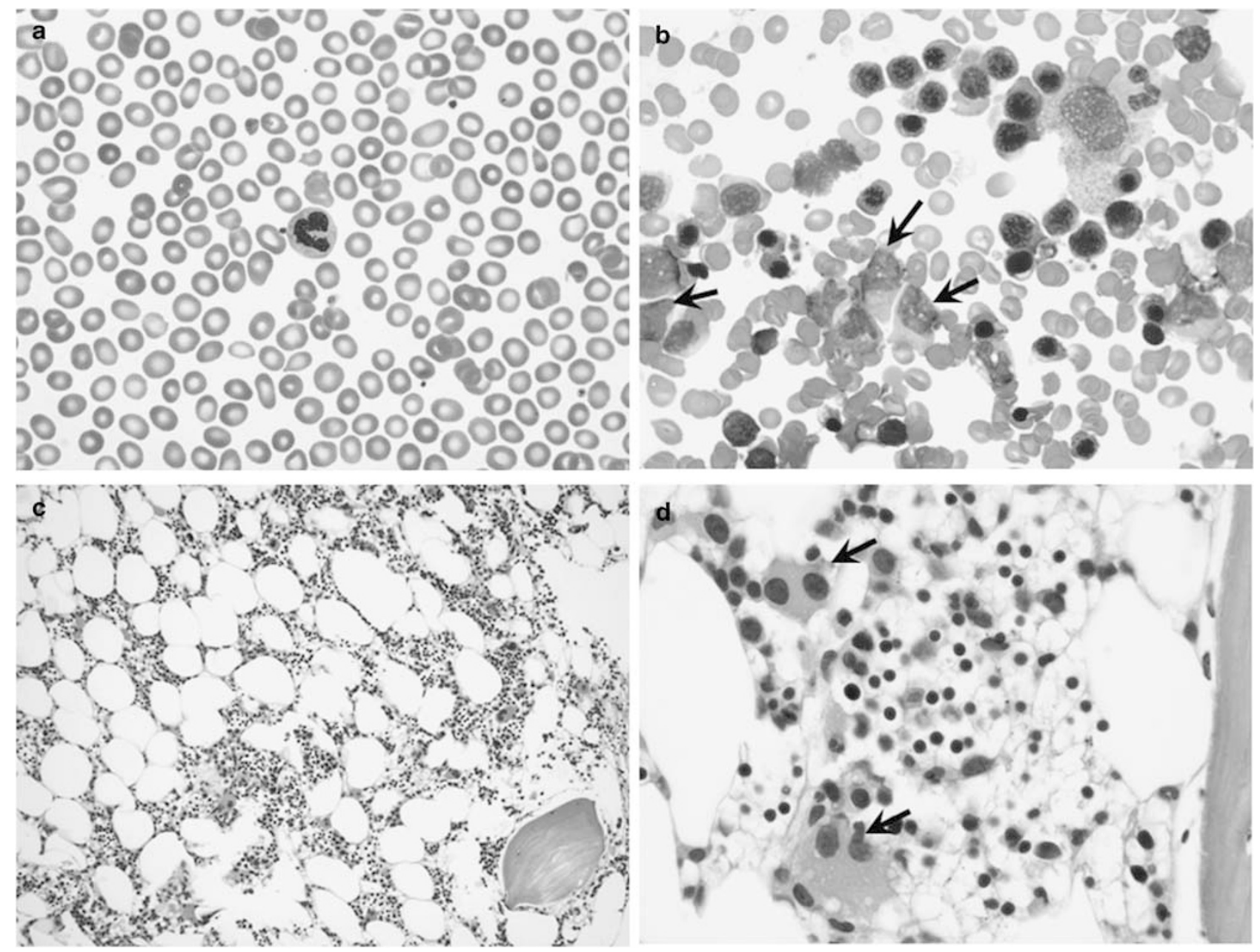

Figure 2 Representative morphological features of therapy-related myelodysplasia arising in chronic lymphocytic leukemia patients after FCR therapy. (a) Peripheral blood smear showing leukopenia $(\times 500)$. (b) Bone marrow aspirate $(\times 1000)$ showing erythroid hyperplasia, dyserythropoiesis, and modestly increased blasts (arrows). (c) Bone marrow biopsy $(\times 100)$ showing a cellularity of $30-40 \%$ and $(\mathbf{d})$ dysplastic megakaryocytes $(\times 400)$ (arrows).

secondary to frontline FCR therapy. This cohort of patients received no previous cytotoxic therapy, and the diagnosis of therapy-related myeloid neoplasms was confirmed by a thorough pathology review, cytogenetic correlation, and clinical followup. This study allows us to estimate the frequency of therapy-related myeloid neoplasms secondary to FCR frontline therapy and to better characterize the clinicopathological and cytogenetic features of therapy-related myeloid neoplasms arising in this clinical setting.

The crude incidence of therapy-related myeloid neoplasms after frontline FCR therapy in this study was estimated from three well-controlled trials: overall $4.5 \%$ with a median follow-up length of 44 months. This number seemed to be lower than that previously reported for up to $19.5 \%$ in patients with indolent lymphoma who received FCR therapy. ${ }^{13-16}$ The difference may be largely attributable to the fact that patients in our study received FCR as the only chemotherapy, whereas most patients in previously published studies (ranging from 67 to $100 \%$ )

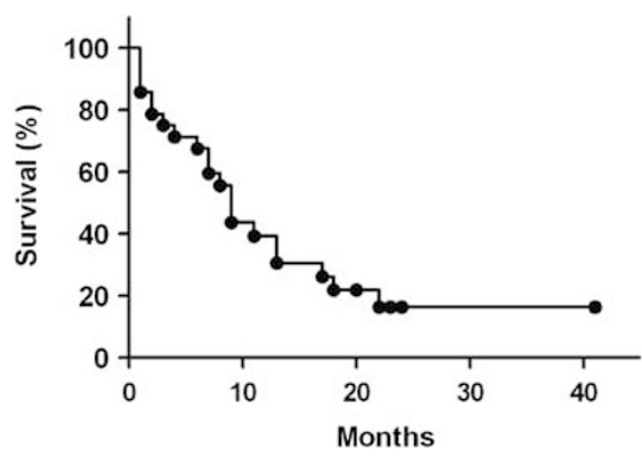

Figure 3 The outcomes of patients who developed therapyrelated myeloid neoplasms after FCR treatment. Patient survival was estimated by the Kaplan-Meier curve from the date of therapy-related myeloid neoplasms diagnosis until death attributed to therapy-related myeloid neoplasms. The median survival was 7 months.

received various alkylating agents before FCR salvage therapy. Therefore, the frequency of therapyrelated myeloid neoplasms in the previous studies likely reflected a combined chemotherapy effects. 
Tam et $a l^{14}$ have suggested that patients who received previous chemotherapy or radiation therapy had higher risk of therapy-related myeloid neoplasms than did patients without previous treatment. Carney and colleagues ${ }^{20}$ also reported similar findings in their study on therapy-related myeloid neoplasms secondary to fludarabine combination therapy in patients with indolent lymphomas. In addition, we observed that the crude incidence of therapy-related myeloid neoplasms after FCR frontline treatment varied, ranging from 1.9 to $8.3 \%$. The therapy-related myeloid neoplasms risk was significantly higher in patients older than 60 years and likely in patients who received GM-CSF co-administration, although the latter was not statistically significant because of small sample size. Interestingly, this higher risk of therapy-related myeloid neoplasms in the elderly was in parallel to a higher incidence of myelotoxicity secondary to FCR occurring in the elderly population. ${ }^{5}$ Reduced hematopoietic stem cell self-renewal and replication capacity in the bone marrow of the elderly may be related to susceptibility to myelotoxicity. GM-CSF may facilitate bone marrow stem cell recovery and reduces risk of infection, but it also allows neoplastic cells to gain growth advantage. Prolonged growth factor treatment has been shown to facilitate selection of neoplastic clone and have a role in the development of myelodysplastic syndrome or acute myeloid leukemia. ${ }^{18,19,21}$

In this study, we confirmed that therapy-related myeloid neoplasms after FCR therapy have morphological characteristics and cytogenetic abnormalities similar to therapy-related myeloid neoplasms caused by typical alkylating agents, evidenced by a more frequent therapy-related myelodysplastic syndrome rather than by therapy-related acute myeloid leukemia presentation, and frequent unbalanced loss of chromosome 5 and/or 7, or a complex karyotype. However, therapy-related myeloid neoplasms associated with FCR have a shorter latency interval, with median 35 months in our series, as compared with 5-7 years generally known for therapy-related myeloid neoplasms secondary to typical alkylating agents. ${ }^{1}$ We observed that a subset of therapy-related myeloid neoplasms could directly emerge from 'persistent myelosuppression' after completion of FCR treatment. The median latency interval in these patients was 23 months, significantly shorter than patients who achieved complete hematological recovery after FCR. Fludarabine, a purine analog, is a potent single agent in chronic lymphocytic leukemia/small lymphocytic lymphoma treatment, ${ }^{22}$ but is also known to cause severe cytopenia more frequently than other chemotherapy agents in chronic lymphocytic leukemia/small lymphocytic lymphoma treatment. ${ }^{22,23}$ This side effect is largely attributed to its cytotoxicity on hematopoietic stem cells. $^{24,25}$ Despite potent myelosuppression, therapyrelated myeloid neoplasms secondary to fludarabine single-agent treatment is extremely rare, reported 0 in $174(0 \%)$ and 1 in $188(0.5 \%)$ in two studies conducted on chronic lymphocytic leukemia/small lymphocytic lymphoma patients who received fludarabine as initial therapy with a long-term followup. $^{26,27}$ However, the incidence of therapy-related myeloid neoplasms is significantly higher in the setting of fludarabine combination therapy. ${ }^{13-16,26,28}$ It has been postulated that fludarabine inhibits DNA repair $^{29}$ and acts synergistically with DNA-damaging agents, such as cyclophosphomide. As a result, genetically altered stem cells may escape DNA integrity check and gain survival advantage in the setting of genotoxic therapy. Impaired immunesurveillance due to fludarabine lymphocyte toxicity also has an important role in the development of therapy-related secondary malignancy. ${ }^{30}$

Myelosuppression is a common side effect of FCR, ${ }^{5,7}$ which can lead to prolonged cytopenia and prompt a bone marrow biopsy. However, persistent cytopenia due to therapy-related myeloid neoplasms is relatively rare. Bone marrow assessment after FCR or cytotoxic therapy in general can be diagnostically challenging, especially in cases in which bone marrow blasts are not increased or cellularity is low. Chemotherapy can cause bone marrow damage and dysplastic changes in hematopoietic cells, even transient cytogenetic abnormalities, ${ }^{31}$ closely mimicking therapy-related myelodysplastic syndrome. These changes can be further complicated by growth factor administration and persistent/ relapsed chronic lymphocytic leukemia/small lymphocytic lymphoma infiltrate in the bone marrow, or concomitant autoimmune cytopenia. We have observed some patients who developed prolonged cytopenia up to 2 years after FCR treatment and were at one point considered as therapy-related myeloid neoplasms, but only declared later. Therefore, the estimation of therapyrelated myeloid neoplasm frequency after FCR treatment can be falsely high if these reversible changes are misinterpreted. In addition, myelodysplastic syndrome might precede or concomitantly exist in some patients at the time of FCR treatment, and only a careful review of patient chart would help to define the myelodysplastic syndrome as therapy-related myelodysplastic syndrome vs primary myelodysplastic syndrome. Vice versa, dysplasia in the early phase of therapy-related myelodysplastic syndrome can be very subtle; sometimes the initial clue is abnormal cytogenetic changes. Overall, 4 of 28 cases in this study had very mild morphological dysplasia and were diagnosed by the presence of cytogenetic abnormalities. Nevertheless, our findings indicate that it is prudent to interpret the morphological findings and cytogenetic abnormalities in the context of clinical presentation and long-term follow-up. An abnormal karyotype was observed in $>90 \%$ of therapy-related myeloid neoplasms cases; therefore, diagnosis of low-grade myelodysplastic syndrome arising in a damaged hypocellular bone marrow requires a 
careful correlation with complete blood count, cytogenetics, and bone marrow morphology.

In summary, this is so far the largest case series of therapy-related myeloid neoplasms arising in chronic lymphocytic leukemia/small lymphocytic lymphoma patients after frontline FCR chemotherapy. This study demonstrated that the risk of therapy-related myeloid neoplasms in this setting was approximately $1.9-8.3 \%$, depending on patient's age, degree of myelosuppression, and concurrent use of growth factors. The latency interval of therapy-related myeloid neoplasms after FCR treatment was shorter than the one generally recognized after alkylating agents or ionizing radiation treatment, implicating a possible synergetic effect of fludarabine. In a subset of patients, therapyrelated myeloid neoplasms can directly arise from a clinical picture of prolonged 'myelosuppression' after chemotherapy, indicating necessary bone marrow examination in this clinical setting to rule out therapy-related myelodysplastic syndrome. Morphological diagnosis of therapy-related myelodysplastic syndrome after FCR could be challenging with superimposed post-treatment changes; and correlation with cytogenetics (positive in >90\% cases) is essential not to overinterpret morphological dysplasia after chemotherapy.

\section{Disclosure/conflict of interest}

The authors declare no conflict of interest.

\section{References}

1 Swerdlow SHCE, Harris NL, Jaffe ES, et al. WHO Classification of Tumours of Haematopoietic and Lymphoid Tissues. International Agency for Research on Cancer: Lyon, 2008.

2 Andre M, Mounier N, Leleu X, et al. Second cancers and late toxicities after treatment of aggressive non-Hodgkin lymphoma with the ACVBP regimen: a GELA cohort study on 2837 patients. Blood 2004;103:1222-1228.

3 Verma D, O’Brien S, Thomas D, et al. Therapy-related acute myelogenous leukemia and myelodysplastic syndrome in patients with acute lymphoblastic leukemia treated with the hyperfractionated cyclophosphamide, vincristine, doxorubicin, and dexamethasone regimens. Cancer 2009;115:101-106.

4 Smith SM, Le Beau MM, Huo D, et al. Clinicalcytogenetic associations in 306 patients with therapyrelated myelodysplasia and myeloid leukemia: the University of Chicago series. Blood 2003;102:43-52.

5 Tam CS, O'Brien S, Wierda W, et al. Long-term results of the fludarabine, cyclophosphamide, and rituximab regimen as initial therapy of chronic lymphocytic leukemia. Blood 2008;112:975-980.

6 Keating MJ, O’Brien S, Albitar M, et al. Early results of a chemoimmunotherapy regimen of fludarabine, cyclophosphamide, and rituximab as initial therapy for chronic lymphocytic leukemia. J Clin Oncol 2005;23: 4079-4088.
7 Gill S, Carney D, Ritchie D, et al. The frequency, manifestations, and duration of prolonged cytopenias after first-line fludarabine combination chemotherapy. Ann Oncol 2010;21:331-334.

8 MacCallum S, Groves M, Brass D, et al. Autoimmune pancytopenia following combination chemotherapy for chronic lymphocytic leukaemia. J Clin Pathol 2009;62:468-470.

9 Borthakur G, O’Brien S, Wierda WG, et al. Immune anaemias in patients with chronic lymphocytic leukaemia treated with fludarabine, cyclophosphamide and rituximab-incidence and predictors. Br J Haematol 2007;136:800-805.

10 Murphy PT, Mitra S, O’Donghaile D. Another case of myelodysplasia with monosomy 7 following fludarabine-based chemotherapy. Am J Hematol 2006; 81:473.

11 Martin-Salces M, Canales MA, de Paz R, et al. Treatment-related acute myeloid leukemia with 11q23 translocation following treatment with fludarabine, cyclophosphamide and rituximab. Leuk Res 2008;32:199-200.

12 Park TS, Cheong JW, Song J, et al. Therapy-related myelodysplastic syndrome with der(17)t(12;17)(q13;p13) as a new recurrent cytogenetic abnormality after treatment for chronic lymphocytic leukemia. Leuk Res 2009; 33:1001-1004.

13 Bowcock SJ, Rassam SM, Lim Z, et al. High incidence of therapy-related myelodysplasia and acute leukaemia in general haematology clinic patients treated with fludarabine and cyclophosphamide for indolent lymphoproliferative disorders. Br J Haematol 2006; 134:242-243.

14 Tam CS, Seymour JF, Prince HM, et al. Treatmentrelated myelodysplasia following fludarabine combination chemotherapy. Haematologica 2006;91:1546-1550.

15 Carney DA, Westerman DA, Tam CS, et al. Therapyrelated myelodysplastic syndrome and acute myeloid leukemia following fludarabine combination chemotherapy. Leukemia 2010;24:2056-2062.

16 Niparuck P, Kanoksil W, Chuncharunee S, et al. Therapy-related myelodysplastic syndrome/acute myeloid leukemia following fludarabine therapy for non-Hodgkin lymphoma and chronic lymphocytic leukemia in Thai patients. Leuk Lymphoma 2010;51: 2120-2125.

17 Hallek M, Cheson BD, Catovsky D, et al. Guidelines for the diagnosis and treatment of chronic lymphocytic leukemia: a report from the International Workshop on Chronic Lymphocytic Leukemia updating the National Cancer Institute-Working Group 1996 guidelines. Blood 2008;111:5446-5456.

18 Bessho M, Itoh Y, Kataumi S, et al. A hematological remission by clonal hematopoiesis after treatment with recombinant human granulocyte-macrophage colonystimulating factor and erythropoietin in a patient with therapy-related myelodysplastic syndrome. Leuk Res 1992;16:123-131.

19 Freedman MH, Bonilla MA, Fier C, et al. Myelodysplasia syndrome and acute myeloid leukemia in patients with congenital neutropenia receiving G-CSF therapy. Blood 2000;96:429-436.

20 Czuczman MS, Emmanouilides C, Darif M, et al. Treatment-related myelodysplastic syndrome and acute myelogenous leukemia in patients treated with ibritumomab tiuxetan radioimmunotherapy. J Clin Oncol 2007;25:4285-4292. 
21 Beekman R, Touw IP. G-CSF and its receptor in myeloid malignancy. Blood 2010;115:5131-5136.

22 Rai KR, Peterson BL, Appelbaum FR, et al. Fludarabine compared with chlorambucil as primary therapy for chronic lymphocytic leukemia. N Engl J Med 2000; 343:1750-1757.

23 Leporrier M, Chevret S, Cazin B, et al. Randomized comparison of fludarabine, CAP, and ChOP in 938 previously untreated stage $\mathrm{B}$ and $\mathrm{C}$ chronic lymphocytic leukemia patients. Blood 2001;98:2319-2325.

24 Herbert KE, Morgan S, Prince HM, et al. Stem cell factor and high-dose twice daily filgrastim is an effective strategy for peripheral blood stem cell mobilization in patients with indolent lymphoproliferative disorders previously treated with fludarabine: results of a Phase II study with an historical comparator. Leukemia 2009;23:305-312.

25 Janikova A, Koristek Z, Vinklarkova J, et al. Efficacious but insidious: a retrospective analysis of fludarabineinduced myelotoxicity using long-term cultureinitiating cells in 100 follicular lymphoma patients. Exp Hematol 2009;37:1266-1273.

26 Morrison VA, Rai KR, Peterson BL, et al. Therapyrelated myeloid leukemias are observed in patients with chronic lymphocytic leukemia after treatment with fludarabine and chlorambucil: results of an intergroup study, cancer and leukemia group B 9011. J Clin Oncol 2002;20:3878-3884.

27 Keating MJ, O’Brien S, Lerner S, et al. Long-term follow-up of patients with chronic lymphocytic leukemia (CLL) receiving fludarabine regimens as initial therapy. Blood 1998;92:1165-1171.

28 McLaughlin P, Estey E, Glassman A, et al. Myelodysplasia and acute myeloid leukemia following therapy for indolent lymphoma with fludarabine, mitoxantrone, and dexamethasone (FND) plus rituximab and interferon alpha. Blood 2005;105:4573-4575.

29 Yamauchi T, Nowak BJ, Keating MJ, et al. DNA repair initiated in chronic lymphocytic leukemia lymphocytes by 4-hydroperoxycyclophosphamide is inhibited by fludarabine and clofarabine. Clin Cancer Res 2001;7:3580-3589.

30 Rashid K, Ng R, Mastan A, et al. Accelerated growth of skin carcinoma following fludarabine therapy for chronic lymphocytic leukemia. Leuk Lymphoma 2005;46:1051-1055.

31 Nakagawa Y, Miura K, Yamazaki T, et al. A case of treatment-related myelodysplastic syndrome spontaneously resolved by drug discontinuance. Int J Hematol 2010;91:530-533. 